\title{
Homozygous TBC1D24 mutation in two siblings with familial infantile myoclonic epilepsy (FIME) and moderate intellectual disability
}

\author{
Anne-Lise Poulat ${ }^{a, d}$, Dorothée Ville ${ }^{a, d}$, Julitta de Bellescize ${ }^{b, d}$, \\ Nathalie André-Obadia ${ }^{c, d}$, Pierre Cacciagli ${ }^{e, d}$, Mathieu Milh ${ }^{e, f, d}$, \\ Laurent Villard $^{\mathrm{e}, \mathrm{d}}$, Gaetan Lescag,h,d,*
}

\footnotetext{
a Department of Pediatric Neurology, Groupement Hospitalier Est, Hospices Civils de Lyon, Lyon, France ${ }^{b}$ Epilepsy, Sleep and Pediatric Neurophysiology Dpt., Groupement Hospitalier Est, Hospices Civils de Lyon, Lyon, France

c Department Epilepsy, Sleep and Functional Neurological Explorations, Groupement Hospitalier Est, Hospices Civils de Lyon, Lyon, France

${ }^{\mathrm{d}}$ Neurophysiology and Epilepsy Unit, Neurological Hospital P. Wertheimer, Hospices Civils de Lyon, Lyon, France

e INSERM, GMGF UMR_S 910, 13385, Aix Marseille Université, Marseille, France

f Service de Neurologie Pédiatrique, Hopital de la Timone-Enfants, APHM, Marseille, France

g Department of Medical Genetics, Hospices Civils de Lyon, Lyon, France

h Université Lyon 1, Lyon, France
}

Summary Mutations in the TBC1D24 gene were first reported in an Italian family with a unique epileptic phenotype consisting of drug-responsive, early-onset idiopathic myoclonic seizures. Patients presented with isolated bilateral or focal myoclonia, which could evolve to long-lasting attacks without loss of consciousness, with a peculiar reflex component, and were associated with generalized tonic-clonic seizures. This entity was named "familial infantile myoclonic epilepsy' (FIME). More recently, TBC1D24 mutations have been shown to cause a variable range of disorders, including epilepsy of various seizure types and severity, non-syndromic deafness, and DOORS syndrome.

We report on the electro-clinical features of two brothers, born to first-cousin parents, affected with infantile-onset myoclonic epilepsy. The peculiar epileptic presentation prompted 
us to perform direct sequencing of the TBC1D24 gene. The patients had very early onset of focal myoclonic fits with variable topography, lasting a few minutes to several hours, without loss of consciousness, which frequently evolved to generalized myoclonus or myoclonic status. Reflex myoclonia were noticed in one patient. Neurological outcome was marked by moderate intellectual disability. Despite the high frequency of seizures, repeated EEG recordings showed normal background rhythm and rare interictal spikes and waves. We found a homozygous missense mutation, c.457G>A/p.Glu153Lys, in the two affected brothers. This observation combined with recent data from the literature, suggest that mutations in TBCD24 cause a pathological continuum, with FIME at the "benign" end and severe drug-refractory epileptic encephalopathy on the severe end. Early-onset myoclonic epilepsy with focal and generalized myoclonic seizures is a common characteristic of this continuum.

(c) 2015 Elsevier B.V. All rights reserved.

\section{Introduction}

Mutations in the TBC1D24 gene (OMIM 613577) have been first reported in an Italian family with autosomal recessive inheritance, including several patients affected with a homogeneous phenotype that was named by the authors: "familial infantile myoclonic epilepsy" (FIME, OMIM 605021) (Falace et al., 2010). The detailed electroclinical presentation, reported ten years earlier, has long remained an isolated description (de Falco et al., 2001). In the past year, thanks to the use of whole-exome sequencing, additional mutations in TBC1D24 have been reported in an increasing range of developmental disorders, including epilepsy of various severity, non-syndromic deafness and DOORS syndrome (OMIM 220500) that gets its name from the acronym of the main features: deafness, onychodystrophy, osteodystrophy, mental retardation, and seizures (Corbett et al., 2010; Milh et al., 2013; Guven and Tolun, 2010; Rehman et al., 2014; Campeau et al., 2014; Stražišar et al., 2014).

In the present paper, we report on two brothers presenting with early-onset myoclonic epilepsy with spontaneous and reflex myoclonia predominant in the face, frequent myoclonic status, moderate developmental delay, and normal interictal EEG, or showing only few paroxysmal abnormalities. On the basis of this particular phenotype, we performed focused genetic analysis to TBC1D24.

\section{Patients and methods}

\section{Patients}

The parents (I-1 and I-2) were healthy first cousins of Algerian origin. There was no additional family history of epilepsy or other neurodevelopmental disorders. The kinship is composed by two affected (II-2 and II-3) boys and two healthy girls (II-1 and II-4). The pedigree of the family is shown in Fig. $1 \mathrm{~A}$.

\section{Patient II-2}

This 10-year-old boy was born in Algeria, at term, by Csection. Birth parameters were normal. Epilepsy started at $24 \mathrm{~h}$ of life with frequent episodes of rapid eyelid myoclonia. Neurological examination revealed global hypertonia and incessant crying. He had daily myoclonic seizures involving the face and the eyelids, sometimes extending to the limbs or the whole body. There was no obvious precipitating factor. Myoclonia occurred daily, in clusters or in status episodes, refractory to valproate, carbamazepine, lamotrigine, phenobarbital, and partially controlled with phenytoin. Prolonged seizures could only be stopped by intravenous clonazepam. Repeated EEG recordings showed a preserved background rhythm and multiple diffuse spikes and slow waves. Cerebral MRI, at the age of 11 months, was normal.

When he was first referred to our neuropediatrics unit, at age of 3 years and 8 months, he had daily myoclonic seizures with variable topography and weekly episodes of generalized myoclonic seizures, without loss of consciousness, which lasted several hours and required high doses of benzodiazepines. One EEG recording, several hours after a prolonged myoclonic seizure that started in the left upper limb, showed a basic theta rhythm of $6-7 \mathrm{~Hz}$. There were neither light-sensitive seizures nor EEG photoparoxysmal response. No ictal EEG could be performed. He had moderate developmental delay without episodes of regression. Sitting was achieved at 9 months and walking at 2 years. He could say a few single words. He could not eat by himself. He had self-injurious behavior and sleep disturbance. He had microcephaly $(48 \mathrm{~cm},-2.5 \mathrm{SD})$ and a large bulbous nose with flat nasal root. There were no finger abnormalities (Fig. 2).

At 6 years and 10 months, he had a cardiac arrest during an Epstein-Barr virus infection, followed by prolonged myoclonic status (treated with clonazepam, phenobarbital and phenytoin) that led to anoxic-ischemic neurological sequelae. Brain MRI, one month later, showed hyperintensity of the lentiform nuclei, moderate ventricular dilatation, and white matter rarefaction, on the T2 and FLAIR sequences. Auditory and visual evoked potentials and electroretinogram were normal. Metabolic screening including lactate/pyruvate, blood and urinary amino acid, creatine and guanidoacetate, transferrin electrophoresis, cerebral spinal fluid analysis, and mitochondrial respiratory chain analysis in muscle were normal. Array-CGH and molecular screening of POLG gene did not show any anomaly.

At last follow-up, the control of myoclonic seizures was mostly achieved with phenytoin, levetiracetam, topiramate, clonazepam, and vitamins (B1, B2, B6, and biotin). Myoclonic status persisted only during febrile episodes. We observed daily erratic myoclonia, triggered by acoustic stimuli. The patient had spastic tetraparesis and moderate 


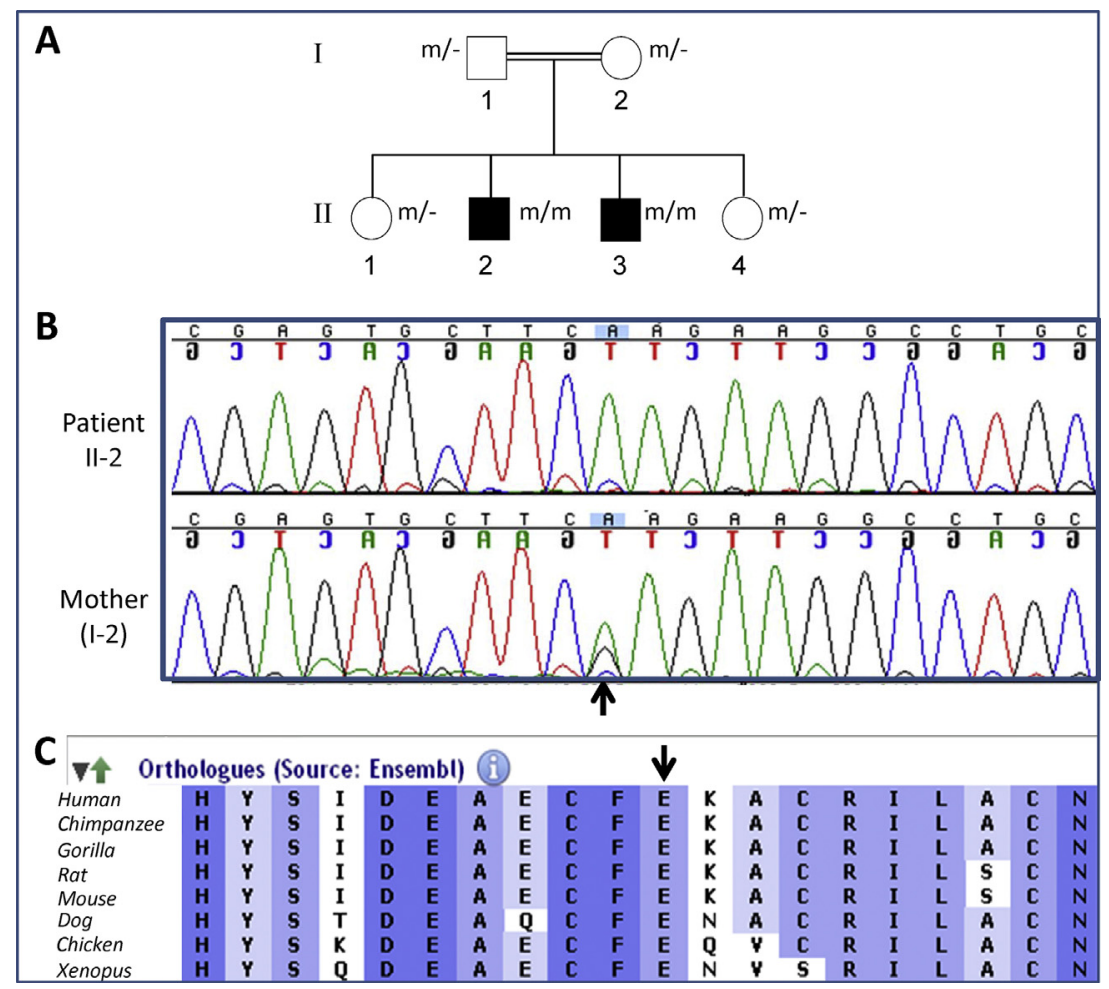

Figure 1 Pedigree and genetic findings. (A) Pedigree of the present family. $m=$ mutation, $-=$ normal allele. (B) Electropherogram showing the C.457G>A mutation (arrow) in patient II-2 (homozygous) and the mother (heterozygous). (C) Local alignment of the TBC1D24 protein sequence among different species showing high conservation of the mutated glutamine.
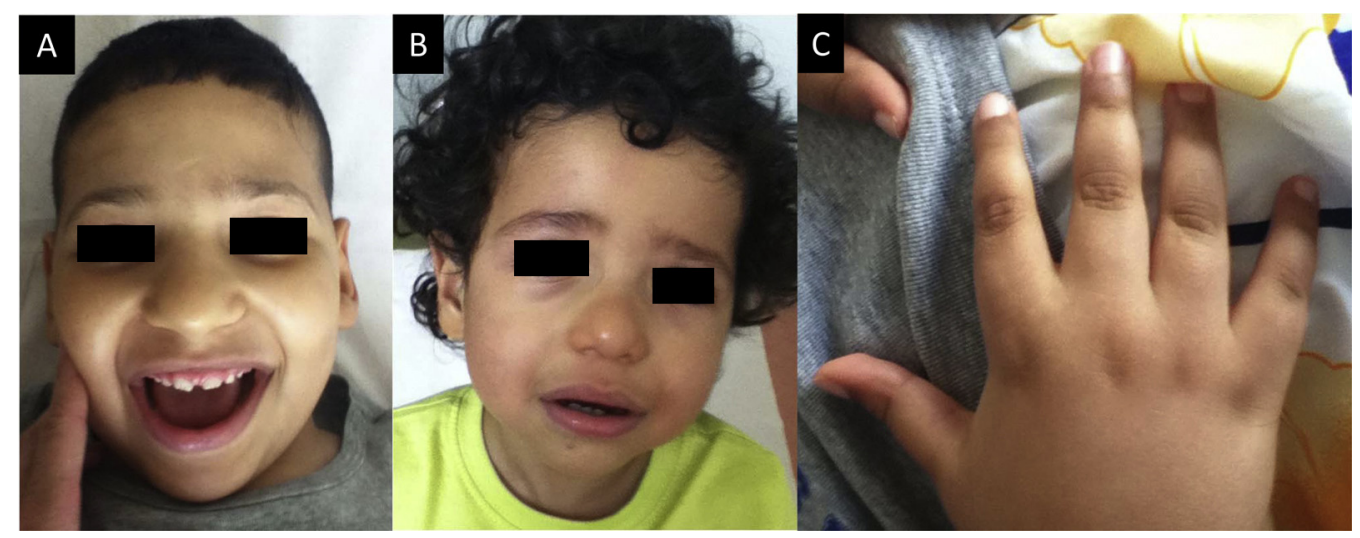

Figure 2 Morphological features of patient II-2 and II-3. (A, B) Flat nasal root and bulbous nasal tip in patients II-2 (A) and II-3 (B). Patient II-3 also has down-slanting palpebral fissures. (C) Right hand of patient II-2 with normal fingers and nails.

intellectual disability. He had no verbal language but had minimal social interaction with a yes-no code. EEG showed a moderate and bilateral slowing of the background rhythm, 6-Hz theta and slow spikes on the vertex, as well as spike and waves in central regions.

\section{Patient II-3}

This 3.5-year-old male patient was born in France at 37 weeks of gestation, by C-section. Birth weight was $3140 \mathrm{~g}$ (mean), height was $52 \mathrm{~cm}$ (mean), and head circumference was $35.5 \mathrm{~cm}$ (mean). Hyperexcitability was noticed soon after birth, characterized by episodes of opistothonus, startles, and incessant crying. Neonatal EEG was normal. Myoclonic epilepsy started at 2.5 months of life. The patient had long-lasting diffuse or generalized myoclonic seizures and daily focal myoclonic episodes of variable topography, involving the eyelids, the peri-oral region, or the whole face, without loss of consciousness. Facial myoclonia were increased by feeding. Myoclonic seizures occurred in clusters, lasting from a few minutes to several hours, with seizure-free intervals not longer than 15 days, exacerbated by febrile episodes. Interictal EEG recordings were normal at age of 2.5 and 3 months. A $24 \mathrm{~h}$-EEG recording, performed at 4 months of life failed to record a status. An ictal recording 


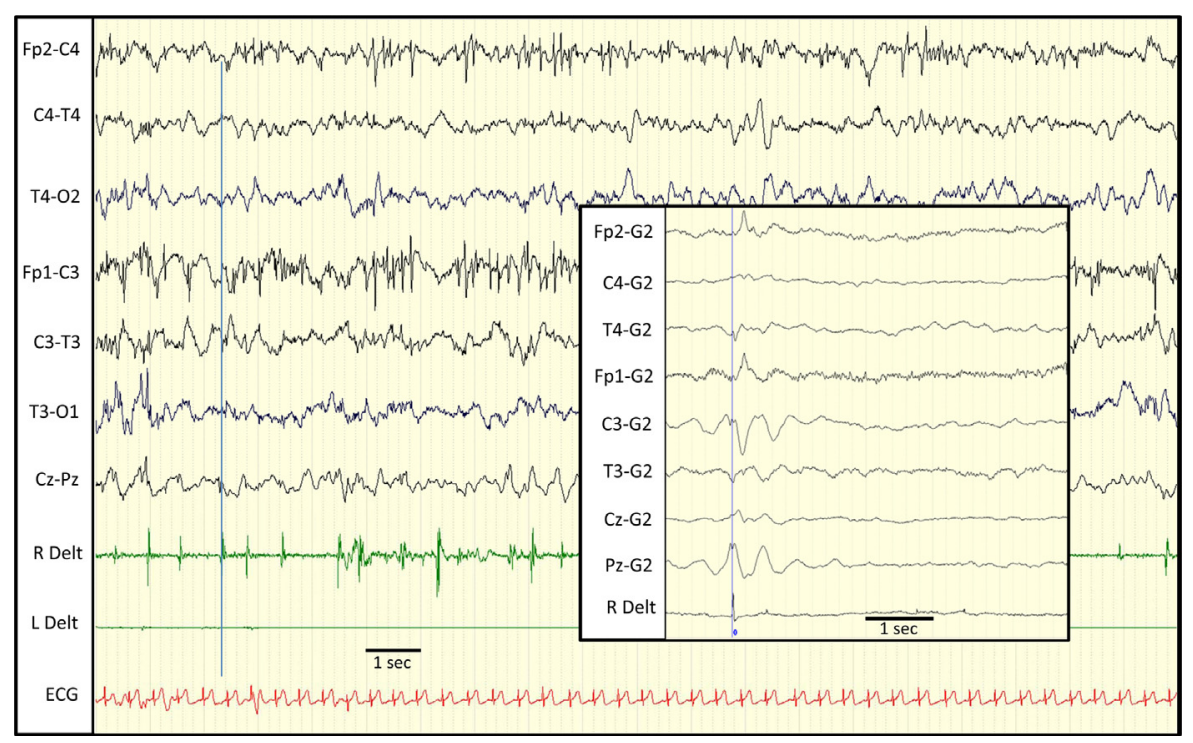

Figure 3 EEG recording of patient 2. Myoclonic partial status involving predominantly the right arm (R Delt: right deltoid). Some of the myoclonic jerks are time locked to low voltage vertex spikes and waves seen on vertex (artifacts in frontal central regions). Framed traces (right panel): EEG back-averaging of 196 right deltoid myoclonic jerks shows that myoclonus is preceded by a cortical potential maximal at $\mathrm{C} 3$ derivation.

was finally obtained several days later: the patient had a 20 min long myoclonus episode of the right arm, associated with sporadic myoclonia that extended to the head, the chest, and the other limbs. Seizures stopped spontaneously during sleep. Ictal EEG was characterized by very low amplitude spikes, mostly on the vertex. At the end of the status, left hemispheric slow waves, predominating in central region, increased and were intermixed with rare spikes and waves (Fig. 3). Jerk-locked back averaging was performed to confirm the cortical origin of myoclonus (Fig. 3). The patient was treated with clonazepam and vitamins (B6, B1, biotin, and pyridoxal phosphate). Phenytoin was added at the age of 4 months, providing partial control of seizures. Since then, he has been having an average of 4 myoclonic status per year.

At 3.5 years of life, he had frequent spontaneous facial myoclonia. Weight was $13.5 \mathrm{~kg}$ (mean), height was $91.5 \mathrm{~cm}$ (mean), and head circumference was $51 \mathrm{~cm}(+1 D S)$. He had moderate developmental delay. He could walk with help and say 10 words. He had a bulbous nose with flat nasal root. He had no hand or foot anomaly and no deafness. Global organization of the interictal wake and sleep EEG was normal, except for rare posterior slow spikes and increased amplitude of the posterior regions, during drowsiness. Brain MRIs, performed at 3 and 23 months, were normal. Metabolic screening including biotinidase, pipecolic acid, lactate/pyruvate, blood and urinary amino acid, urinary organic acid, and neurotransmitters dosage in CSF were normal.

\section{Mutation analysis}

Informed consent for genetic analyses was obtained from the parents, according to the French bioethics law. Sanger sequencing of the eight exons and intronic junctions of the TBC1D24 gene was performed in patient II-2 (Genbank reference sequence: NM_001199107). In silico predictions of pathogenicity was performed using the Alamut Software (Interactive biosoftware, Mont Saint-Aignan, France). The segregation of the mutation with the phenotype was performed in DNA from the parents and siblings.

\section{Results}

The c.457G >A missense substitution, located in exon 2, was found at the homozygous state in patient II-2 and in patient II-3 (Fig. 1B). This substitution led to the replacement of a glutamate, highly-conserved through evolution, to a lysine, p.Glu153Lys (Fig. 1C). It was predicted to be diseases causing by SIFT (score: 0 ) and Polyphen-2 (score: 1) softwares. The parents and the two sisters were heterozygous carriers of the mutation (Fig. $1 \mathrm{~A})$. This mutation has been reported in a single heterozygous carrier $(1 / 6437$ genotypes) in the Exome Variant server database (http://evs.gs.washington.edu/EVS/) and in 10 individuals from the Exome Aggregation Consortium database (http://exac.broadinstitute.org/), which gives a frequency around $10^{-5}$ in the control population.

\section{Discussion}

The two brothers reported here had an unusual epileptic disease characterized by very early onset of focal myoclonic fits, lasting a few minutes to several hours, without loss of consciousness that regularly evolved to generalized myoclonus or myoclonic status. In patient II-3, facial myoclonia were triggered by feeding. In contrast, neurological outcome was marked by moderate intellectual impairment with no regression, and only few paroxysmal abnormalities on standard interictal EEGs. The more severe neurological impairment of patient II-2 was related to the 
anoxic brain injury. We could confirm the cortical origin of myoclonus by jerk-locked back averaging. The electroclinical constellation observed in these patients was very similar to the peculiar phenotype, described as FIME, except for moderate cognitive impairment (Berg et al., 2010).

Ten years after having mapped the FIME locus to chromosome 16p13.3, mutations in the TBC1D24 gene have been found by targeted next-generation sequencing (Falace et al., 2010; de Falco et al., 2001). Despite the recognition of FIME as a specific electro-clinical entity among the classification of the International League Against Epilepsy (ILAE), other families with a similar phenotype have been rarely reported.

We performed direct sequencing of TBC1D24 in our patients because they shared a significant number of phenomenological aspects with those from the former FIME family, such as early onset myoclonic epilepsy, with high seizure frequency and variable topography of myoclonic seizures, affecting hands, legs, eyelids, and eyeballs, which could evolve to diffuse myoclonia or myoclonus status. In our patients, a reflex component was present and interictal EEG showed only mild paroxysmal abnormalities, as in the original FIME family (Falace et al., 2010).

Additional mutations of TBC1D24 have been reported recently, thanks to exome sequencing in patients with $a$ priori unrelated disorders. Several patients had a more severe neurological presentation (Corbett et al., 2010; Milh et al., 2013; Duru et al., 2010; Stražišar et al., 2014). Corbett et al. reported a large consanguineous family with seven affected individuals, who were described as having focal seizures with prominent eye blinking, as well as facial and limb jerking, which started at two months of age and persisted throughout life (Corbett et al., 2010; Afawi et al., 2013). Those patients had mild-to-moderate intellectual disability. EEG data were not reported. Other mutations in TBC1D24 have been found in three families with devastating infantile epileptic encephalopathy (Milh et al., 2013; Duru et al., 2010; Stražišar et al., 2014). In the first one, the patients had long-lasting myoclonic seizures that were exacerbated by common infections and that did not respond to medication, with neurological deterioration (Duru et al., 2010). EEG recordings in the initial stages were characterized by multiple spikes that later evolved to progressive slowing of background activity. The electro-clinical presentation of the two affected sisters from the second family was consistent with the diagnosis of malignant migrating partial seizures of infancy (Milh et al., 2013). In the third family, the epileptic phenotype was very similar to the patients reported here despite very severe neurological outcome, with early lethality in one patient (Stražišar et al., 2014).

Mutations of TBC1D24 were recently shown to be the major cause of DOORS syndrome (Campeau et al., 2014). Although our two patients share some dysmorphic features with patients affected with DOORS syndrome, they lack the other features constitutive of this syndrome. A variety of seizure types have been observed in patients with DOORS syndrome, including generalized tonic-clonic, complex partial focal clonic seizures, and infantile spasm (Campeau et al., 2014). Although precise description of the epileptic features was not available in that series, a myoclonic presentation was mentioned in more than $20 \%$ of the patients.
In myoclonic epilepsy related to TBC1D24 mutations, myoclonia seem to involve more frequently the face, and especially the peri-orbicular and peri-oral muscles, as well as the upper limbs, suggesting an increased cortical excitability affecting more selectively the Rolandic area. TBC1D24 belongs to the TBC domain containing proteins, highly conserved through evolution, and is a regulator of neurite length and branching (Falace et al., 2010; Corbett et al., 2010; Campeau et al., 2014). Highest expression levels of Tbc1d24 in mice brain tissue were seen in the somatomotor and auditory areas, and in the hippocampal formation (Allen Brain Atlas: http://mouse.brain-map. org/experiment/show?id=69531049). Mutations in TBC1D24 may lead to hyperexcitability of the Rolandic region, which generates cortical myoclonus. The p.Glu153Lys mutation is located within the TBC domain of the protein, close to the p.Asn147His mutation that was found (compound heterozygous with the p.Ala515Val mutation) in the former FIME family, and distinct from those found in patients with more severe phenotypes (Falace et al., 2010). This may suggest that missense mutations of TBC1D24 may have different functional consequences according their location on the protein sequence. It is also possible that truncating mutations may cause a more severe course of disease, with high mortality rate (Guven and Tolun, 2010; Stražišar et al., 2014).

The two patients from the present family had an electroclinical presentation consistent with the diagnosis of FIME. Our patients, however, had more severe cognitive impairment and myoclonia were resistant to treatment. This observation, combined to recent data from the literature suggest that mutations in TBCD24 cause a pathological continuum, with FIME at the "benign"' end and severe drugrefractory encephalopathy on the severe end. Early-onset myoclonic epilepsy with focal and generalized myoclonic seizures is the most common feature of this continuum.

\section{Acknowledgements}

We thank the patients and parents for their participated to the study.

\section{References}

Afawi, Z., Mandelstam, S., Korczyn, A.D., Kivity, S., Walid, S., Shalata, A., Oliver, K.L., Corbett, M., Gecz, J., Berkovic, S.F., Jackson, G.D., 2013. TBC1D24 mutation associated with focal epilepsy, cognitive impairment and a distinctive cerebrocerebellar malformation. Epilepsy Res. 105, 240-244.

Berg, A.T., Berkovic, S.F., Brodie, M.J., Buchhalter, J., Cross, J.H., van Emde Boas, W., Engel, J., French, J., Glauser, T.A., Mathern, G.W., Moshé, S.L., Nordli, D., Plouin, P., Scheffer, I.E., 2010. Revised terminology and concepts for organization of seizures and epilepsies: report of the ILAE Commission on Classification and Terminology, 2005-2009. Epilepsia 51, 676-685.

Campeau, P.M., Kasperaviciute, D., Lu, J.T., Burrage, L.C., Kim, C., Hori, M., Powell, B.R., Stewart, F., Félix, T.M., van den Ende, J., Wisniewska, M., Kayserili, H., Rump, P., Nampoothiri, S., Aftimos, S., Mey, A., Nair, L.D., Begleiter, M.L., De Bie, I., Meenakshi, G., Murray, M.L., Repetto, G.M., Golabi, M., Blair, E., Male, A., Giuliano, F., Kariminejad, A., Newman, W.G., Bhaskar, S.S., Dickerson, J.E., Kerr, B., Banka, S., Giltay, J.C., Wieczorek, D., Tostevin, A., Wiszniewska, J., Cheung, S.W., Hennekam, R.C., Gibbs, R.A., Lee, B.H., Sisodiya, S.M., 2014. The genetic 
basis of DOORS syndrome: an exome-sequencing study. Lancet Neurol. 13, 44-58.

Corbett, M.A., Bahlo, M., Jolly, L., Afawi, Z., Gardner, A.E., Oliver, K.L., Tan, S., Coffey, A., Mulley, J.C., Dibbens, L.M., Simri, W., Shalata, A., Kivity, S., Jackson, G.D., Berkovic, S.F., Gecz, J., 2010. A focal epilepsy and intellectual disability syndrome is due to a mutation in TBC1D24. Am. J. Hum. Genet. 87, 371-375.

de Falco, F.A., Majello, L., Santangelo, R., Stabile, M., Bricarelli, F.D., Zara, F., 2001. Familial infantile myoclonic epilepsy: clinical features in a large kindred with autosomal recessive inheritance. Epilepsia 42, 1541-1548.

Duru, N., Iseri, S.A.U., Selcuk, N., Tolun, A., 2010. Early-onset progressive myoclonic epilepsy with dystonia mapping to 16pterp13.3. J. Neurogenet. 24, 207-215.

Falace, A., Filipello, F., La Padula, V., Vanni, N., Madia, F., De Pietri Tonelli, D., de Falco, F.A., Striano, P., Dagna Bricarelli, F., Minetti, C., Benfenati, F., Fassio, A., Zara, F., 2010. TBC1D24, an ARF6-interacting protein, is mutated in familial infantile myoclonic epilepsy. Am. J. Hum. Genet. 87, 365-370.
Guven, A., Tolun, A., 2010. TBC1D24 truncating mutation resulting in severe neurodegeneration. J. Med. Genet. 50, 199-202.

Milh, M., Falace, A., Villeneuve, N., Vanni, N., Cacciagli, P., Assereto, S., Nabbout, R., Benfenati, F., Zara, F., Chabrol, B., Villard, L., Fassio, A., 2013. Novel compound heterozy-gous mutations in TBC1D24 cause familial malignant migrating partial seizures of infancy. Hum. Mutat. 34, 869-872.

Rehman, A.U., Santos-Cortez, R.L., Morell, R.J., Drummond, M.C., Ito, T., Lee, K., Khan, A.A., Basra, M.A., Wasif, N., Ayub, M., Ali, R.A., Raza, S.I., University of Washington Center for Mendelian Genomics, Nickerson, D.A., Shendure, J., Bamshad, M., Riazuddin, S., Billington, N., Khan, S.N., Friedman, P.L., Griffith, A.J., Ahmad, W., Riazuddin, S., Leal, S.M., Friedman, T.B., 2014. Mutations in TBC1D24, a gene associated with epilepsy, also cause nonsyndromic deafness DFNB86. Am. J. Hum. Genet. 94, 144-152.

Stražišar, B.G., Neubauer, D., Paro Panjan, D., Writzl, K., 2014. Early-onset epileptic encephalopathy with hearing loss in two siblings with TBC1D24 recessive mutations. Eur. J. Paediatr. Neurol. S1090-3798, 212-218. 